\title{
A hydraulic model to simulate the hydrodynamics of a fluorine electrolyser
}

\author{
J. P. Caire ${ }^{1}$, G. Espinasse ${ }^{2}$, M. Dupoizat ${ }^{3} \&$ M. Peyrard ${ }^{4}$ \\ ${ }^{I}$ LEPMI, Grenoble INP, France \\ ${ }^{2}$ BERTIN Technologies, France \\ ${ }^{3}$ AREVA Business Unit Chimie, \\ Secteur Mines Chimie Enrichissement, France \\ ${ }^{4}$ ASTEK Rhône-Alpes, France
}

\begin{abstract}
Fluorine electrolysis is characterized by very large overpotentials and bubble effects that are not yet fully understood. The two-phase free flows appearing in the fluorine reactor are complex and attributable mainly to hydrogen bubbles evolving at the cathode. However, large fluorine bubbles gliding along the anode help to drag the electrolyte up along the anode and in doing so also take part to the two-phase movement. The fluorine electrolyser has been modelled in the past but there has been no means of comparing hydraulic computations with measurements in such an aggressive environment. A hydraulic model is presented here to test the ability of the Estet-Astrid finite volume code to model the fluorine reactor. The two-phase free flow was modelled using an Euler-Euler scheme assuming bubbles of uniform diameter. Laser Particle Image Velocimetry was used to measure both gas and liquid velocities. This paper presents the experimental study and the model made to obtain the plume shape. Numerical and experimental results are compared and some discrepancies are explained. Improvements are suggested for future modelling
\end{abstract}

Keywords: hydrogen, fluorine, electrolysis, two-phase flow, hydraulic model, free convection, Estet-Astrid.

\section{Fluorine electrolysis}

Fluorine electrolysis is characterized by very large overpotentials and bubble effects $[1,2]$. The fluorine electrolyser has been extensively described in [3]. The 
characteristics of fluorine bubble release are not yet completely understood [1, 4]. The two-phase free flow is mainly due to hydrogen bubbles evolving at the cathode, but large fluorine bubbles also take part in the two-phase movement, as seen in fig. 1a. H. Roustan [1] has shown that to some extent the large gliding fluorine bubbles drag the electrolyte up along the anode. A numerical model was gradually developed in France to account for all the strongly linked phenomena involved in fluorine electrolysis [2-6]. The final numerical model combined two commercial codes, Flux-Expert (FE) and Estet-Astrid (EA), both distributed by Astek [7, 8]. Estet-Astrid is a CFD finite volume code for computing the free convection of two-phase flows. In the present study it was used with an EulerEuler model. The Estet-Astrid code took into account the forces acting on individual bubbles, namely buoyancy, drag force, lift force and added mass. Thanks to EA, Espinasse et al. estimated the fraction of the hydrogen plume that recombines with fluorine and thereby decreases Faraday current efficiency in an industrial fluorine electrolyser [6].
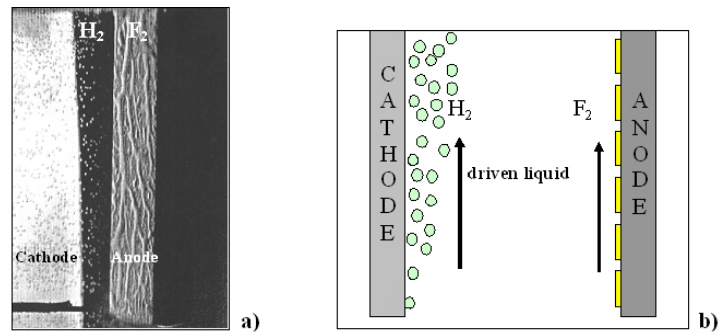

Figure 1: a) Fluorine and hydrogen bubbles. b) Equivalent scheme of hydrogen plume and fluorine bubbles.

Since the plume of hydrogen bubbles is so important in a fluorine electrolyser, experimental work was carried out to validate the accuracy of plume prediction by EA. KF-2HF molten salt electrolyte is so aggressive that it makes laser Particle Image Velocimetry (PIV) measurements difficult in a large fluorine cell. We therefore designed a hydraulic set-up using water (see fig. 3) in order to simulate the two-phase hydrodynamics of the fluorine electrolyser. We present the specific experimental study performed to determine the shape of the plume and the EA computations. The experimental and CFD results are then compared.

Hydrogen bubbles generated in fluorine electrolysis are very small (diameter close to $250 \mu \mathrm{m}$ ). Fluorine behaves in an unusual way: fluorine bubbles slip along the anode instead of detaching from the electrode in the same way as hydrogen bubbles. Fig. 1b) shows a schematic representation of the plume of hydrogen and the bubbles of fluorine that slip along the electrode, partially coated with CFx [4]. The hydrodynamics of a fluorine electrolyser are completely atypical. Roustan [1] showed that free convection of KF-2HF electrolyte was induced by both the hydrogen plume and the slipping film of fluorine, as shown in fig. 2. Electrolyte velocity was measured in the vicinity of the anode by PIV thanks to small alumina beads placed in a laboratory cell. 
Fig. 2 shows that velocity increases with height in an almost linear way along the cylindrical anode at low current density. With an increase in current density, i.e. in the fluorine flow rate on the anode, the velocity increases up to a plateau [1].

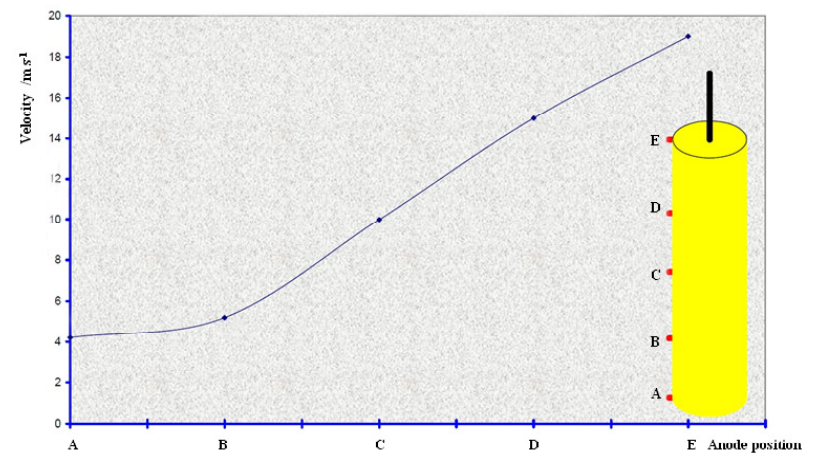

Figure 2: $\quad$ Fluid velocity in vicinity of a cylindrical anode in a laboratory fluorine cell.

This drag effect was interpreted as follows: the almost continuous film of fluorine bubbles adhering to the anode slips upwards increasingly quickly and moves the electrolyte in its vicinity. Because of the large gas cover rate on the anode, the local current density is extremely high and the bubbles interact with the electrolyte, according to us, when small liquid jets exchange mass between the electrode and the bulk in a way similar to that described in Higbie's penetration model [9]. Thus, the film of fluorine bubbles acts as a mobile wall that moves the liquid roughly at its speed (fig. 2). This idea of a moving wall was used in the hydraulic model. The hydrogen bubbles evolving at the cathode are driven by the molten salt and in the absence of a separator between the anode and cathode compartments (no diaphragm is resistant enough for use in a fluorine electrolyser) they can come in contact with fluorine and recombine spontaneously into hydrofluoric acid. Espinasse et al. [6] showed that when the intensity of the cell increases, Faraday efficiency decreases because of this recombination.

It is thus particularly important to accurately simulate the hydrodynamics of the fluorine cell so as to predict its operation correctly. KF-2HF molten salt at $95{ }^{\circ} \mathrm{C}$ is very corrosive and extremely delicate to handle. It was thus decided to build a hydraulic model using water and nitrogen bubbles, which is easier to study and presents fewer hazards, the final objective being to validate the EA numerical model used previously for the fluorine cell.

\section{Hydraulic model}

The 60x60x10 cm scale model made out of Plexiglas is shown in fig. 3. Nitrogen flowing through a gas sparger fitted with a circular sintered-glass filter created a fairly realistic plume of nitrogen bubbles, probably similar to the hydrogen 


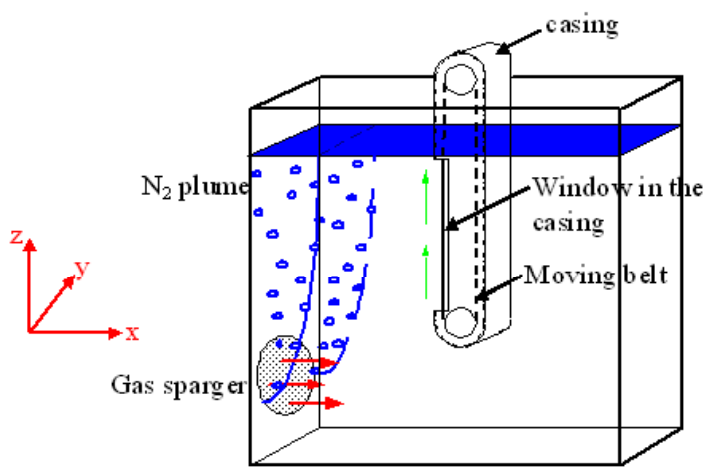

Figure 3: Scheme of the hydraulic model with the 3 axis directions used in this study.

plume in the fluorine cell. The behaviour of the fluorine in the linear part of fig. 2 was simulated by a notched belt moving at constant speed which sweeps water along in the tank just as fluorine drags the electrolyte in the fluorine cell. The belt was covered with a partially opened casing to simulate an equivalent anode; the flow was therefore channelled only in the vertical direction. The belt casing and its associated electric motor could be moved along the $\mathrm{x}$-axis to visualize the influence of the spacing between the two pseudo-electrodes. The model is not very thick for two reasons: i) PIV cannot be used to measure velocity along the $y$-axis, ii) free convection movements in the vertical plane are favoured, though Fig. 6 shows that some small parasitic movements appear in the y-direction. Thus it can be estimated that the fluorine film creeping along the anode surface is conveniently represented by the moving belt.

\section{Measurements}

Particle Image Velocimetry (PIV) was used to determine the velocity vectors in the tank. The method is based on the image processing of a laser light sheet created in the vertical z-x plane which allows measurement of the displacement of $14 \mu \mathrm{m}$ metallised hollow glass beads carried by the flow. Nitrogen flow was injected between 0 and $100 \mathrm{l} / \mathrm{h}$ through a circular sintered-glass filter. Ethylene glycol was added to modify the viscosity of the fluid from 1 to $3.4 \mathrm{mPa}$.s and decrease the diameter of the nitrogen bubbles from 5 to $1 \mathrm{~mm}$, value close to the estimated hydrogen bubbles diameter in the fluorine reactor [13]. The uniform linear speed of the belt could be varied between 0 and $0.3 \mathrm{~m} / \mathrm{s}$ to simulate in some extent the fluorine behaviour.

\subsection{Study of bubble plume alone}

Since the PIV system could retrieve only one signal at a time for each phase it was not possible to obtain simultaneous measurements of both liquid and 
nitrogen bubble velocities in the plume. Thus, the velocity vectors were obtained successively through the left window of fig. 4 for the nitrogen bubbles in the plume and through the right window for the liquid.

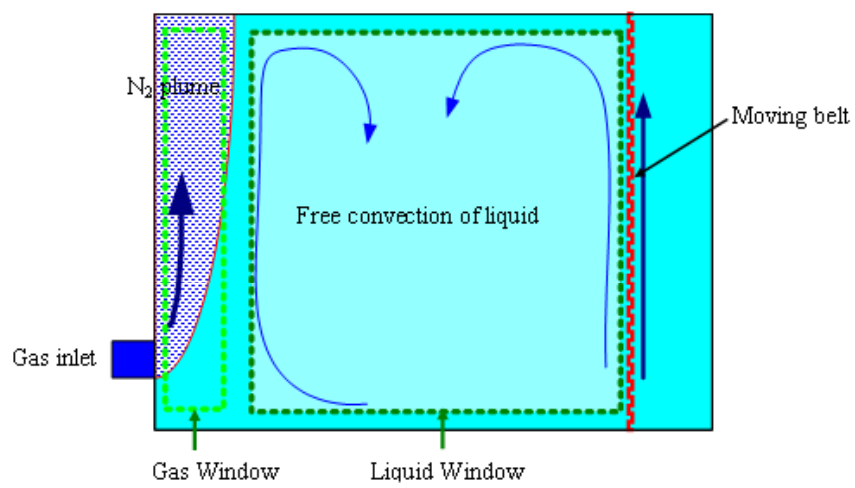

Figure 4: The two windows used for measuring gas and liquid velocity vectors in z-x plane.
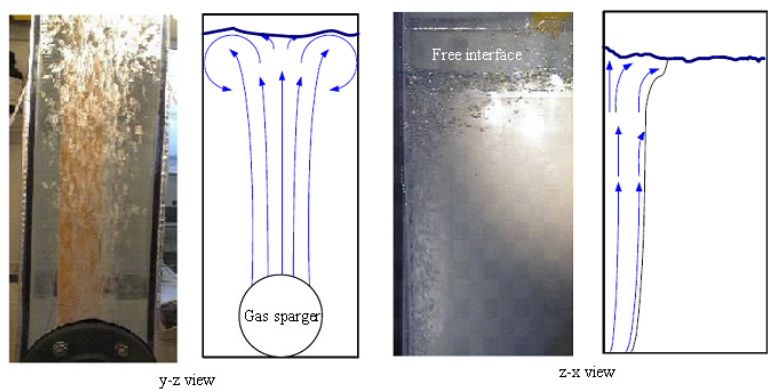

Figure 5: $\quad$ Plume of nitrogen bubbles and schematic movements of fluids in the $\mathrm{y}-\mathrm{z}$ and $\mathrm{z}-\mathrm{x}$ directions (bubble diameter $5 \mathrm{~mm}$ - gas flow rate $50 \mathrm{l} / \mathrm{h})$.

Fig. 5 shows the bubble plume in front view ( $y-z$ plane) and profile ( $\mathrm{z}-\mathrm{x}$ plane). In both cases the photo is accompanied by a drawing schematizing the observed flows. For a constant gas input, the total gas/liquid contact surface is greater for small bubbles than for larger bubbles and the gas-liquid interactions are also greater. It was observed that the smaller the bubbles, the greater the liquid velocity, the more agitated and more turbulent the bubble plume, and the more spread out the plume at the air/liquid interface.

\subsection{Study of the notched belt alone}

The speed of the notched belt could be changed to visualize its convective effect on the liquid initially at rest. Fig. 6 shows a photo of the liquid, with the moving 
belt seen at left. The velocity vectors computed from PIV measurements were superimposed on the photo. The picture is of the upper part of the tank taken when the movement of the liquid was instigated by the belt alone.

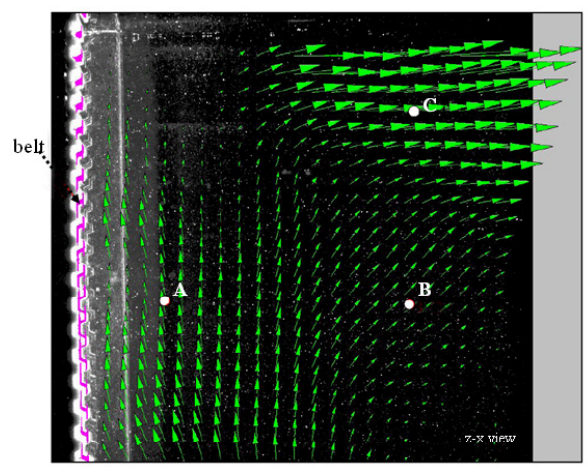

Figure 6: Photo of liquid circulated by the notched with the reference points A, B, C.

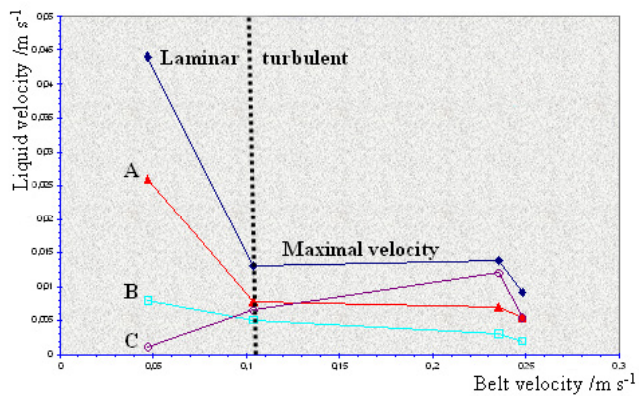

Figure 7: $\quad$ PIV velocity of liquid circulated by the notched belt alone.

Fig. 7 presents the liquid velocities measured at A, B, C (see fig. 6) when the belt alone is actuated. A laminar/turbulent transition appears at a belt speed of $0.1 \mathrm{~m} / \mathrm{s}$. For speeds higher than $0.1 \mathrm{~m} / \mathrm{s}$, the flow becomes turbulent, and the liquid also moves in the third dimension i.e. along y-axis, as seen in fig. 5-left. In turbulent conditions, water is no longer dragged at the belt speed, and the slip velocity becomes significant. A Reynolds number could be assigned to the belt:

$$
\operatorname{Re}_{\mathrm{b}}=\frac{\mathrm{LV}}{\mathrm{v}}
$$

where $\mathrm{L}$ is the belt width in $\mathrm{m}, \mathrm{V}$ the velocity of the belt in $\mathrm{m} / \mathrm{s}$ and $v$ the kinematic viscosity in $\mathrm{m}^{2} / \mathrm{s}$. With this definition, the laminar-turbulent transition was observed at a $\mathrm{Re}_{\mathrm{b}}$ value of 2000 , i.e. for $\mathrm{V}$ equal to $0.1 \mathrm{~m} / \mathrm{s}$. In laminar conditions, the maximum velocities observed in the liquid (see fig. 7) are very 
close to the belt speed. At large distances from the belt and particularly at points $\mathrm{A}, \mathrm{B}$, and $\mathrm{C}$ the influence of the belt is less marked and the liquid velocities are lower. In turbulent conditions, maximum velocity is lower since belt-liquid coupling is lessened and the slip velocity becomes significant.

Similar behaviour was observed for a fluorine film in a small laboratory cell when current density was increased i.e. when the velocity of the fluorine film on the anode was increased [1]. This confirms that the moving belt is a convenient method for simulating the fluorine film.

\section{3D Numerical modelling}

The Estet-Astrid commercial CFD code was used to simulate the hydraulic model. This finite volume code was used with a two-phase Euler-Euler K- $\varepsilon$ turbulence model [6]. In this case the code solved a mass balance equation and a momentum balance equation both for the liquid and the gas. In this model, the liquid is regarded as the continuous phase, while the gas constitutes the minority phase, i.e. the dispersed phase. Compared with a Euler-Lagrange two-phase model, strong coupling between phases is easily treated and transients are more easily computed. The model did not take into account bubble diameter distribution (Log-Normal distributions are often observed in this case). However, Antal et al. [10] have shown that distribution of bubble diameters is the most significant parameter for a good description of plumes. The bubbles are regarded as rigid spheres. The two-phase model took into account as in [6] the following forces exerted on bubbles: 1 - gravity, 2 - buoyancy (Archimedes' force), 3 - the force exerted by the flow on the bubble which results from two terms: the pressure effect, strongly related to the existence of a wake for poorly shaped particles; and the effect of the viscous term that dominates in well shaped bodies, which is the case for a spherical gas bubble. These effects can be split into two forces: a drag force in the direction of incidental flow and a lift force in the direction perpendicular to incidental flow, 4 - added mass, i.e. the force on the liquid related to the particle acceleration.

It can be observed that the bubble diameter comes into play in all these forces at power 2 or 3 , making the computation very sensitive to this parameter. There are many other forces which could be considered (compressive force due to the liquid, surface tension forces, wake-related forces exerted by neighbouring bubbles, electrostatic repulsion forces between bubbles, etc.). However, these forces were not taken into account in this numerical model. The physical data used for the two phases are shown in Table 1.

Table 1: $\quad$ Physical properties of the continuous and disperse phases.

\begin{tabular}{|l|c|c|}
\hline & $\mathbf{H}_{\mathbf{2}} \mathbf{O}$ & $\mathbf{N}_{\mathbf{2}}$ \\
\hline Pressure & $101300 \mathrm{~Pa}$ & $101300 \mathrm{~Pa}$ \\
\hline Density & $998 \mathrm{~kg} \mathrm{~m}^{-3}$ & $1.12 \mathrm{~kg} \mathrm{~m}^{-3}$ \\
\hline Kinematic viscosity & $10^{-6} \mathrm{~m}^{2} \mathrm{~s}^{-1}$ & $2.18 .10^{-5} \mathrm{~m}^{2} \mathrm{~s}^{-1}$ \\
\hline Inlet mass flow rate & $0,0124 \mathrm{~kg} \mathrm{~m}^{-2} \mathrm{~s}^{-1}$ & \\
\hline Bubbles diameter & & $0.001-0.005 \mathrm{~m}$ \\
\hline
\end{tabular}




\subsection{Initial conditions}

Initially the water was assumed to be at rest and the gas fraction null at any point of the system. At initial time, the gas (made up of bubbles of diameter d) was introduced at a constant mass flow rate with a given speed. The problem was thus time-dependent, and therefore solved in non-stationary conditions until stationary conditions were reached. The solution then perfectly described the transient evolution of the gas plume.
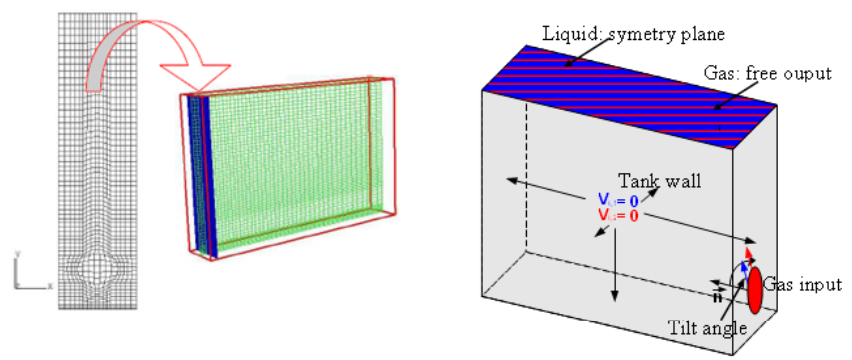

Figure 8: $\quad$ Boundary conditions and curvilinear structured meshing.

\subsection{Boundary conditions}

Fig. 8 describes the boundary conditions used here for a plume of nitrogen composed of 1 millimetre-diameter bubbles. At gas output, the free surface of the water was assumed to be constantly at rest and the normal liquid velocity vectors null:

$$
\frac{\partial \mathrm{V}}{\partial \mathrm{n}}=0
$$

On this symmetry plane, the boundary condition for pressure is as follows:

$$
\frac{\partial^{2} \mathrm{P}}{\partial \tau \partial \mathrm{n}}=0
$$

This classical condition of EA [8] describes the ideal free liquid surface and let the gas pass freely through it. At walls of the tank the velocities were assumed to be null for the two phases. At gas input, a uniform velocity profile was imposed for the dispersed phase. It was assumed here that the input velocity vectors were tilted at $45^{\circ}$ upwards from the horizontal in order to obtain realistic hydraulic 
conditions. This arbitrary condition was required since convergence problems appeared when the bubbles were injected perpendicularly to the sintered-glass filter.

\subsection{Grid mesh}

Fig. 8 shows the structured curvilinear grid used in these calculations. The grid related to the hydraulic model is made of 18 planes in x-direction, 59 in $\mathrm{y}$ direction, 45 in z-direction and 47790 nodes. No special meshing law was required at tank wall since a no slip condition was imposed in EA [8]. To describe the formation of plume which fully develops in 2 seconds it was necessary to use a time step close to $10^{-5}$ second, requiring a computing time of approximately 70 hours on an INTEL bi Xeon $2.5 \mathrm{GHz}$ PC. Fig. 9 presents the velocities calculated by EA for gas bubbles and liquid along AB. The bubble velocity and liquid velocity increase with height. Both velocities have a similar profile on $\mathrm{AB}$, but the bubble velocity is much higher than that of the liquid. The difference, close to $0.38 \mathrm{~m} / \mathrm{s}$, is attributed to the slip velocity between bubbles and liquid. This value is much more realistic than the $1.3 \mathrm{~m} / \mathrm{s}$ free rising velocity of bubbles calculated from the Stokes formula.

\section{Comparison of experimental and numerical results}

The comparison between measurements and numerical results of fig. 10 shows some disparities. Three zones can be distinguished. In zone 1 , close to the sparger at the plume bottom, the diameter of the bubbles is gauged at less than 1 $\mathrm{mm}$. Along the plume, the diameter of the rising bubbles increases, probably by coalescence. Since all the forces acting on the bubbles depend on their diameter, the numerical model overestimates the size of the bubbles in the bottom of the plume. In the second zone, computed and measured velocities are practically identical. It is thought that the bubble diameters have their nominal value in this zone.

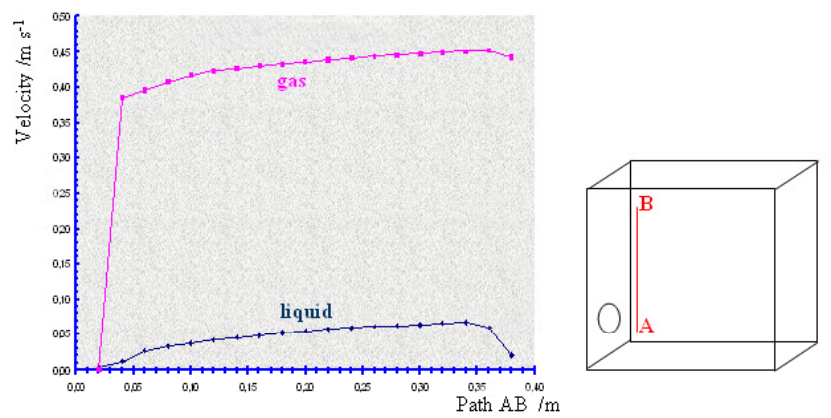

Figure 9: $\quad$ Velocity computed on path $\mathrm{AB}$ situated at $2 \mathrm{~cm}$ from the left wall.

The third zone is at the top of tank where the plume spreads out. The numerical model again overestimates the gas velocity, probably because the 
effect of the interface on escaping bubbles was not taken into account in this model. Closer examination of the bubble plume in fig. 11-left reveals that the bubble plume widens along the air-water interface whereas the computed plume seen in fig. 11-right is very narrow in this area.
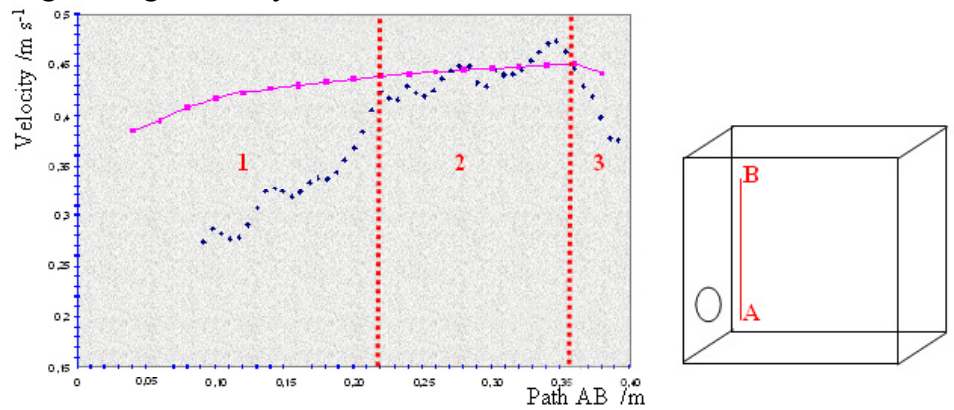

Figure 10: Comparison between numerical model and measurements for gas velocity on $\mathrm{AB}$.

The difference between model and calculation undoubtedly comes from the effect of the free interface, which was not taken into account here. This model also lacks the interactions between bubbles: in reality, the rising bubbles collide with each other and then lose kinetic energy. The numerous collisions between quasi-rigid bubbles probably explain the widening of the plume seen in fig. 11 . Moreover, the numerical model did not take into account the interactions that might exist between the bubbles in the plume and those at the sparger outlet. Nor did the model take into account bubble diameter distribution. This poor description of a plume is not related to EA.
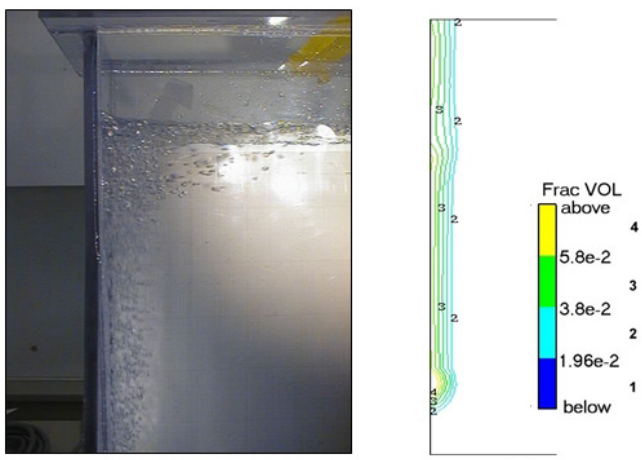

Figure 11: $\quad$ Photo of nitrogen plume and computed volume fraction of gas.

None of the usual commercial codes satisfactorily describes this type of plume, as underlined by Antal et al. [10] when comparing the three commercial codes CFX, FLUENT and NPHASE with the same plume benchmark. The main 
issues are probably related to the lack of interactions between bubbles and their environment (other bubbles, wakes of other bubbles, walls, air-liquid interface). Antal et al. [10] have also pointed out that it is not realistic to assume uniform diameters since diameter distributions of bubbles usually follow a Log-Normal law [13]. These problems are encountered in many fields [11], and particularly in the modelling of electrochemical reactors [11-12]. It is thus necessary to take account of these observations in order to improve models of two-phase fluids and obtain more realistic bubble plumes.

\section{Conclusions}

A hydraulic model giving a vertical two-phase flow similar to that of a fluorine cell was designed and investigated. Experimental results confirmed the similarities in behaviour between the hydraulic model and the fluorine cell. The comparison of numerical and PIV results for the hydraulic model showed that gas and liquid velocity vectors were realistic and of the same order of magnitude. The results were in good agreement for the nominal diameter of the bubbles and discrepancies occurred when coalescence was visible. The belt used as a moving wall behaved in a similar way to the gliding film of fluorine bubbles on an anode. However, the discrepancies observed - particularly for the gas plume reflected the lack of interactions between bubbles in this model. Moreover, the model required a long computing time due to the very small time step necessary to properly describe the transient flows in the tank and attain the stationary state. Further modelling work is in progress within the framework of the AMELHYFLAM project to improve the hydrodynamics of fluorine reactors.

\section{Acknowledgements}

The authors are grateful to AREVA and the French project AMELHYFLAM (ANR 2007) for their financial support.

\section{References}

[1] Roustan H., Modélisation des transferts couplés de charge et de chaleur dans un électrolyseur industriel de production de fluor, INPG thesis, Grenoble, 1998.

[2] Roustan H., Caire J.P., Nicolas F. \& Pham P., Modelling coupled transfers in an industrial fluorine electrolyser. Jal of Applied Electrochemistry, 28, pp. 237-243, 1998.

[3] Nicolas F., Techniques de l'Ingénieur, J 6 325, Editions T.I., Paris.

[4] Groult H., Devilliers D., Lantelme F. \& Caire J.P., Combel M., Nicolas F., Origin of the anodic overvoltage observed during fluorine evolution in KF-2HF, J. of Elelectrochem. Soc., 149, E485-E492, 2002.

[5] Roustan H., Caire J.P., Nicolas F. \& Pham P., in J.W. Van Zee, T.F. Fuller, P.C. Foller and F. Hine (Eds), 'Advances in Mathematical 
Modeling and Simulation of Electrochemical Processes', Electrochem. Soc. Proc. USA 98-10, 202, 1998.

[6] Espinasse G., Peyrard M., Nicolas F., Caire J.P., Effect of hydrodynamics on Faradaic current efficiency in a fluorine electrolyser, Jal of Applied Electrochemistry 37, 77-85, 2007.

[7] Flux-Expert, Guide de l'Utilisateur, ASTEK, Paris, 2001.

[8] Estet-Astrid, Guide de l'Utilisateur, ASTEK, Paris, 2001.

[9] Higbie R., The rate of absorption of pure gas into a still liquid during short periods of exposure. Transactions of A.I.Ch.E. 31 365, 1935.

[10] Antal, S.P., Ettorre, S.M., Kunz, R.F., Podowski, M.Z., Development of a next generation computer code for the prediction of multicomponent multiphase flows. In: proceeding of the International Meeting on Trends in Numerical and Physical Modeling for Industrial Multiphase Flow, Cargese, France, 2000.

[11] Mandin P., Hamburger J., Bessou S. \& Picard G., Modelling and calculation of the current density distribution evolution at vertical gasevolving electrodes, Electrochemica Acta, 51, pp.1140-1156, 2005.

[12] Jomard J., Feraud J.P., Morandini J., Du Terrail Couvat Y. \& Caire J.P., Hydrogen filter press electrolyser modelled by coupling Fluent and Flux Expert codes, Jal of Applied Electrochemistry, 38, pp. 297-308, 2008.

[13] Caire J.P., unpublished results. 